\title{
To Evaluate the Efficacy and Safety of Autologous Platelet Rich Fibrin Matrix in the Treatment of Non-Healing Ulcer of Various Aetiology
}

\author{
Bhansali Shreyansh ${ }^{1}$, Mehta Mahaveer ${ }^{2}$, and Kachhawa Dilip ${ }^{3 *}$ \\ ${ }^{1}$ Senior resident, Department of Dermatology, Dr SN Medical College, Jodhpur, India \\ ${ }^{2}$ Chairman and Medical Director, Dr Mahaveer Mehta Medical centre, Dubai, UAE \\ ${ }^{3}$ Senior professor and HOD, Department of Dermatology, Dr SN Medical College, Jodhpur, India
}

*Corresponding author: Dilip K, Senior professor and HOD, Department of Dermatology, Dr SN Medical College, Jodhpur, India, Tel: 9414130036; E-mail: drdilipkachhawa@hotmail.com

Received: 20 Jan, 2020 | Accepted: 14 Feb, 2020 | Published: 20 Feb, 2020

Citation: Shreyansh B, Mehta M, Dilip K (2020) To Evaluate the Efficacy and Safety of Autologous Platelet Rich Fibrin Matrix in the Treatment of Non-Healing Ulcer of Various Aetiology. J Clin Cosmet Dermatol 4(2): dx.doi.org/10.16966/2576-2826.147

Copyright: (C) 2020 Shreyansh B, et al. This is an open-access article distributed under the terms of the Creative Commons Attribution License, which permits unrestricted use, distribution, and reproduction in any medium, provided the original author and source are credited.

\begin{abstract}
Rationale: Chronic Non-Healing Ulcers (NHU) is those which persists for more than 6 weeks and may be due to underlying factors like venous stasis, peripheral neuropathy, immobility, etc. Chronic ulcers are associated with decreased quality of life and also pose a financial burden on the patient as well as society. The failure of NHU to respond to the various modalities available lead to the search for growth factors requisite for wound healing resulting in the development of Platelet Rich Plasma (PRP). Platelet Rich Fibrin Matrix (PRFM) is a next generation of PRP that has shown promising result in our study.
\end{abstract}

Objectives: This study was done to evaluate the efficacy and safety of autologous platelet rich fibrin matrix in the treatment of non-healing ulcer of various aetiology.

Results: All the ulcers responded to the treatment with decrease in pain and discharge beginning from first sitting. The mean time to ulcer healing was $6.43+2.33$ weeks. There was $100 \%$ improvement in volume at the end of 8 weeks and $96 \%$ improvement in area at the end of 12 weeks.

Conclusion: This study demonstrates that Autologous PRFM is a safe and efficacious method for the treatment of non-healing ulcer and at the same time simple and highly cost effective method.

Keywords: Non-healing ulcer; Platelets; Autologous platelet rich fibrin matrix; Growth factors

Abbreviations: NHU: Non-Healing Ulcer; PRFM- Platelet Rich Fibrin Matrix; GF: Growth Factors

\section{Introduction}

Wound healing is an orderly set of processes culminating in closing the wound, any interference in this process at any step can lead to non-healing and when this process lasts for more than 6 weeks it is termed as a Non-Healing Ulcer (NHU) [1]. Various aetiologies like diabetes, Leprosy, trauma, venous stasis, pyoderma, bed sores, etc., can be the underlying cause of NHU. The morbidities associated like chronic pain, difficulty in walking, sight of NHU and the potential risk of complications make it a very frustrating condition for the patient. In addition the costs involved like frequent visits and stay in hospital, the costs of medicines too add a significant financial burden to the patient. Various existing modalities like placental extract, collagen dressing, surgical debridement, split thickened skin grafting, etc., all suffer from a basic lacunae in the form of lack of growth factors. Newer modalities include hirudotherapy [2], follicular unit extraction [3] and transplant on the wound bed, epidermal grafting, etc. Recently, the focus has shifted on to growth factors which include therapies like recombinant growth factors and Platelet Rich Plasma (PRP). Platelet Rich Fibrin Matrix (PRFM), a next generation PRP is the latest addition which is based on the premise of slow and sustained release of growth factors [4] mimicking the natural wound healing process. The concentration of platelets is higher in PRP but a study showed that, when incorporated into fibrin, the mean concentration of growth factors in the platelet concentrates was three times or more than that observed with conventional platelet-rich plasma. The membrane can be easily placed on the ulcer with minimal chances of displacement. Thus giving PRFM an edge over PRP in wound healing.

Autologous PRFM is prepared from patients own blood thus being free from risk of communicable pathogens, is easy to prepare with long lasting as well as good clinical results. 


\section{Objectives}

The present study was done to evaluate the efficacy and safety of Autologous PRFM in the treatment of NHU of various aetiology.

\section{Methodology}

This is a prospective interventional hospital based study conducted on 25 patients attending the OPD of Department of Dermatology, MDM Hospital, DR SNMC Medical College, Jodhpur with clinically diagnosed chronic non healing ulcers of varied aetiology after applying inclusion criterion. Ethical clearance was taken from Institutional Ethical Committee. Ulcers more than 6 week duration, ulcer having slopping or scarred edges, and Diabetic patients with controlled blood sugar were included in the study. Exclusion criterion include critically ill patients, actively infected ulcer, patients with evidence of underlying osteomyelitis, diabetes with uncontrolled blood sugar, bleeding disorders and psychiatric patients.

After taking an informed consent patient's complete history was elaborated followed by local and systemic examination. Local examination included number, site, size, shape, surface, edge, floor, base of the ulcer, discharge and tenderness, surrounding skin, and regional lymph node examination. Size of the ulcer was measured using the formula for an ellipse: Length $\times$ width $\times 0.7854[5,6]$. Volume was measured using the formula: (Length $\times$ width $\times 0.7854$ ) $\times$ depth. Routine investigations included Haemoglobin, CBC, Platelet count, BT, CT, PT-INR, Random Blood Sugar, Liver function test, Blood Urea and Serum Creatinine, Viral markers: HIV, HBsAg and Urine-Albumin, Sugar, Microscopy, Colour Doppler lower limb was also done wherever required. The patients included in the study were explained about the entire procedure and only after taking written informed consent the procedure was performed.

Supportive treatment including analgesics, oral and topical antibiotics was given to all patients and in case of dirty looking ulcer debridement was done prior to the procedure. Treatment was also directed towards the underlying etiological cause. Diabetic patients were treated with oral hypoglycemic drugs and subcutaneous insulin depending on the blood sugar levels. MDT was continued in patients of leprosy and adequate rest was advised for varicose ulcers. For bed sores, repeated change in position and pressure relieving mattresses were advised.

$10 \mathrm{ml}$ venous blood was taken using a $10 \mathrm{cc}$ syringe under strict aseptic precautions. The blood was transferred to a sterile centrifugation tube devoid of anticoagulant. The tube was than centrifuged at 4000 rpm for ten minutes Centrifugation resulted in formation of three distinct layers with the lowermost layer comprising of red blood cells; the middle layer of platelet rich fibrin membrane and platelet poor plasma formed the upper layer.

Platelet poor plasma was discarded by tilting the tube and using a sterile forceps PRFM was separated from the lowermost layer and transferred to sterile gauze. The ulcer was first cleaned and margin was freshened by dermabrasion, then the membrane was applied and then non-absorbable dressing was done followed by secondary gauze dressing. The patient was started on tablet cefixime $200 \mathrm{mg} \mathrm{BD}$ and during the treatment course adequate rest was assured. After 7 days the dressing was opened and the dried PRFM was removed.

The process was repeated with fresh PRFM every 7 days as per requirement up to maximum of five sittings. At each visit, healing of the ulcer was assessed using area and volume measurements calculated using the formula for an ellipse. Photographic records were also made.
Categorical/nominal variables were summarized as number and percentage and were analyzed using chi square test. Continuous variables were summarized as mean and standard deviation and analyzed using student $\mathrm{t}$-test for intergroup comparison. For comparison of continuous variables in same group at different times, repeated measures ANOVA test was used. A P-value less than 0.05 were taken as statistically significant. All statistical analysis were done using Epi info version 7.2.1.0.

\section{Observation and Results}

25 patients with chronic ulcer of more than 6 weeks duration were treated with PRFM at weekly interval up to a maximum of 5 sittings. Out of 25 patients, 18 (72\%) were males and 7 (28\%) were females. Mean age was $48+11$ years with maximum 10 (40\%) patients belonging to the age group of 40-50 years. In majority, 9 (36\%) patients the underlying aetiology was traumatic followed by varicose veins and diabetes in $3(12 \%)$ patients each, leprosy in $2(8 \%)$ and other causes in remaining.

17 (68\%) patients had ulcer of duration 1.5-2.5 months, 7 (28\%) had since 2.5-4 months and 1 (6\%) patient had ulcer of more than 4 month duration. 20 (80\%) patients had ulcer on lower limb of which 18 were in lower leg and 1 each on upper leg and sole, $3(12 \%)$ had ulcer on abdomen and $2(8 \%)$ patients on upper leg. 9 (36\%) patients reported pain at presentation and $15(60 \%)$ patients gave history of discharge from ulcer. 4 (16\%) patients had hypertension and $6(24 \%)$ had underlying diabetes.

Complete ulcer healing was noted in 23 (92\%) patients while partial healing was observed in $2(8 \%)$ patients. Out of completely healed ulcers, 14 (60.9\%) patients had healing within 4-8 weeks, 7 (30.4\%) ulcers took less than 4 weeks to heal and $2(8.4 \%)$ ulcers healed in 8-12 weeks' time. Overall the mean time to ulcer healing was $6.43+2.33$ weeks (Figures 1-3)

Mean area of ulcer at presentation was $10.95+9.08 \mathrm{~cm}^{2}$. Mean area reduced to $6.44+6.85 \mathrm{~cm}^{2}$ at end of 2 weeks (41.8\% improvement), $3.96+6.11 \mathrm{~cm}^{2}$ at the end of 4 weeks (63.8\% improvement), $2.22+5.24$ $\mathrm{cm}^{2}$ at end of 6 weeks (79.7\% improvement) and $0.43+1.51 \mathrm{~cm}^{2}(96 \%$ improvement) at the end of 12 weeks (Figure.2). At each follow up the improvement in mean area of ulcer was found to be statistically significant. The mean volume of ulcer at presentation was $5.96 \pm 4.49$ $\mathrm{cm}^{3}$ which reduced to $1.96 \pm 1.99 \mathrm{~cm}^{3}$ (67\% improvement) at the end of 2 weeks, $0.42 \pm 1.05 \mathrm{~cm}^{3}$ (92.9\% improvement) at the end of 4 weeks, $0.02 \pm 0.08 \mathrm{~cm}^{3}$ at 6 weeks ( $99.7 \%$ improvement) and became $0(100$ $\%$ improvement) at the end of 8 weeks (Figures 3-5). Thus significant healing of chronic ulcer was observed with this technique. No side effects were noted.

\section{Discussion}

Ulcer is defined as any breach in the continuity of epidermis or mucus membrane. Ulcers can be classified as acute and chronic depending upon the time it persists. Chronic ulcers are those which persist for more than 6 weeks [1] and may be due to underlying factors like venous stasis, peripheral neuropathy, immobility, etc. Chronic ulcers are associated with persistent discharge, chronic pain, difficulty in walking, loss of limb, decrease in quality of life, etc. and are a cause of constant embarrassment for the patient. The treatment is difficult and entails huge costs on the patient as well as the society.

Platelet Rich Plasma (PRP) consists of an increased concentration of autologous platelets 3-5 times that of native plasma $[7,8]$ suspended in a small amount of plasma. Multiple growth and differentiation factors are released from PRP which results in accelerated soft tissue wound healing, 2-3 times faster than that of normal [9]. 

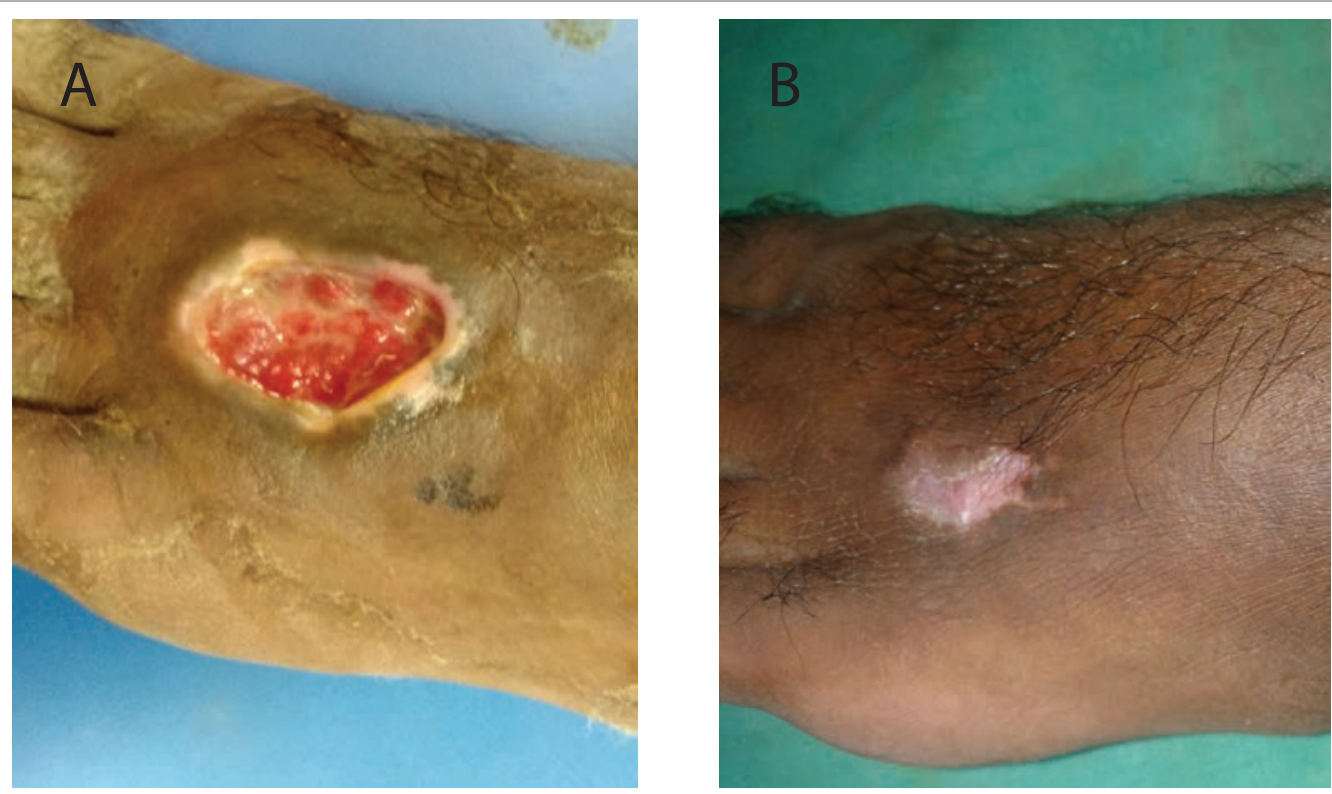

Figure 1: A patient with traumatic ulcer of 4 month duration on dorsal aspect of left foot.

A: Picture taken before the procedure.

B: Completely healed ulcer at the end 10 weeks.
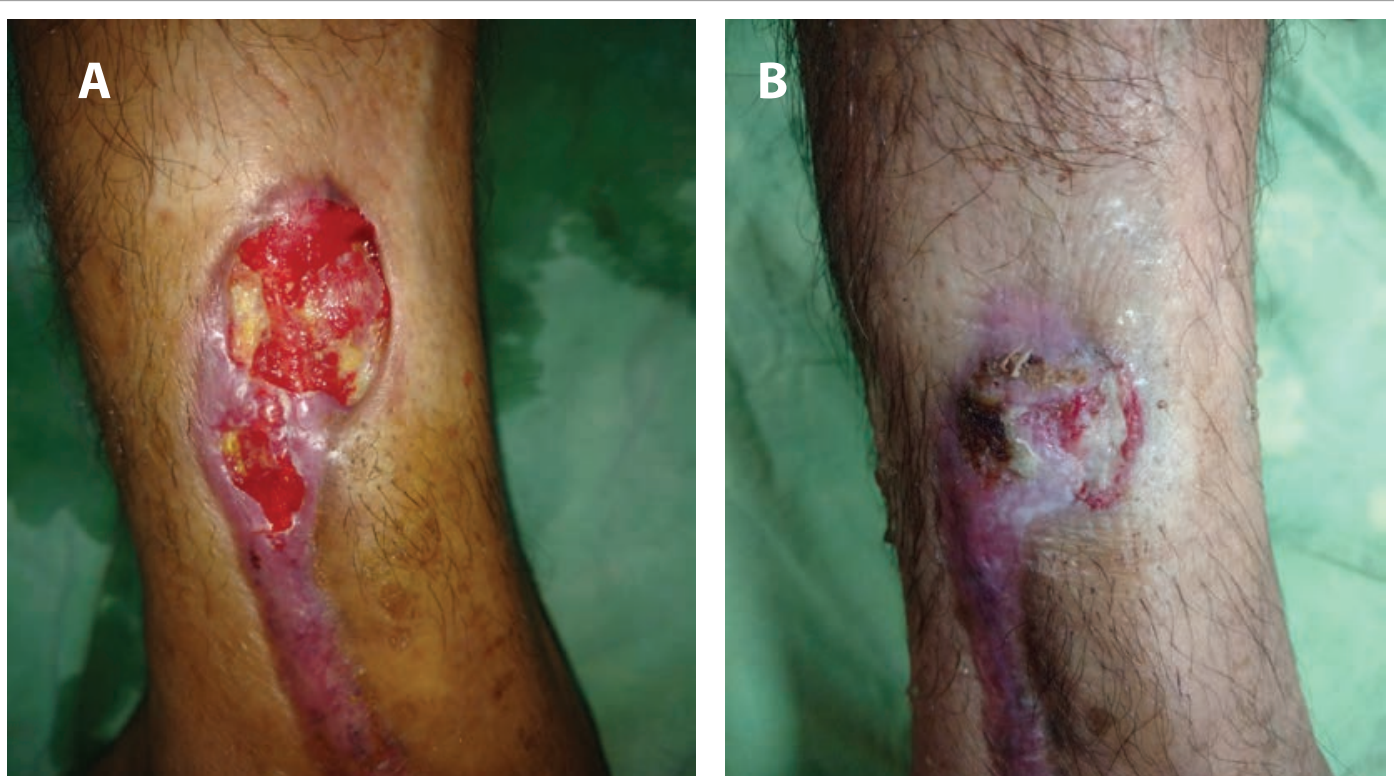

Figure 2: Patient of iatrogenic ulcer on left lower leg developing after surgery for lymphatic disorder.

A: Before the technique.

B: 6 weeks after Autologous prfm.

PRFM is a second generation platelet derivative first developed by Choukroun J, et al. [10] for use in oral and maxillofacial surgery. PRFM is a membrane based preparation of PRP which does not require addition of thrombin [11].

During the process of centrifugation a matrix is formed by the natural polymerisation process, this fibrin Matrix is highly cross linked and is responsible for the real therapeutic potential of PRFM. Ultrastructurally, PRFM shows dense highly cross-linked fibrin matrix with the intact platelets localized on one side of the membrane.
The fibrin matrix present in PRFM is flexible, elastic, and very strong and consists of weak thrombin concentrations which entail equilateral junctions. These junctions help in the formation of a fine and flexible fibrin network that supports platelets and cytokines and cellular migration that occurs.

A study by Yazawa M, et al. [12] showed that, when incorporated into drug delivery systems such as fibrin, the mean concentration of growth factors in the platelet concentrates was three times or more than that observed with conventional PRP and its natural fibrin 

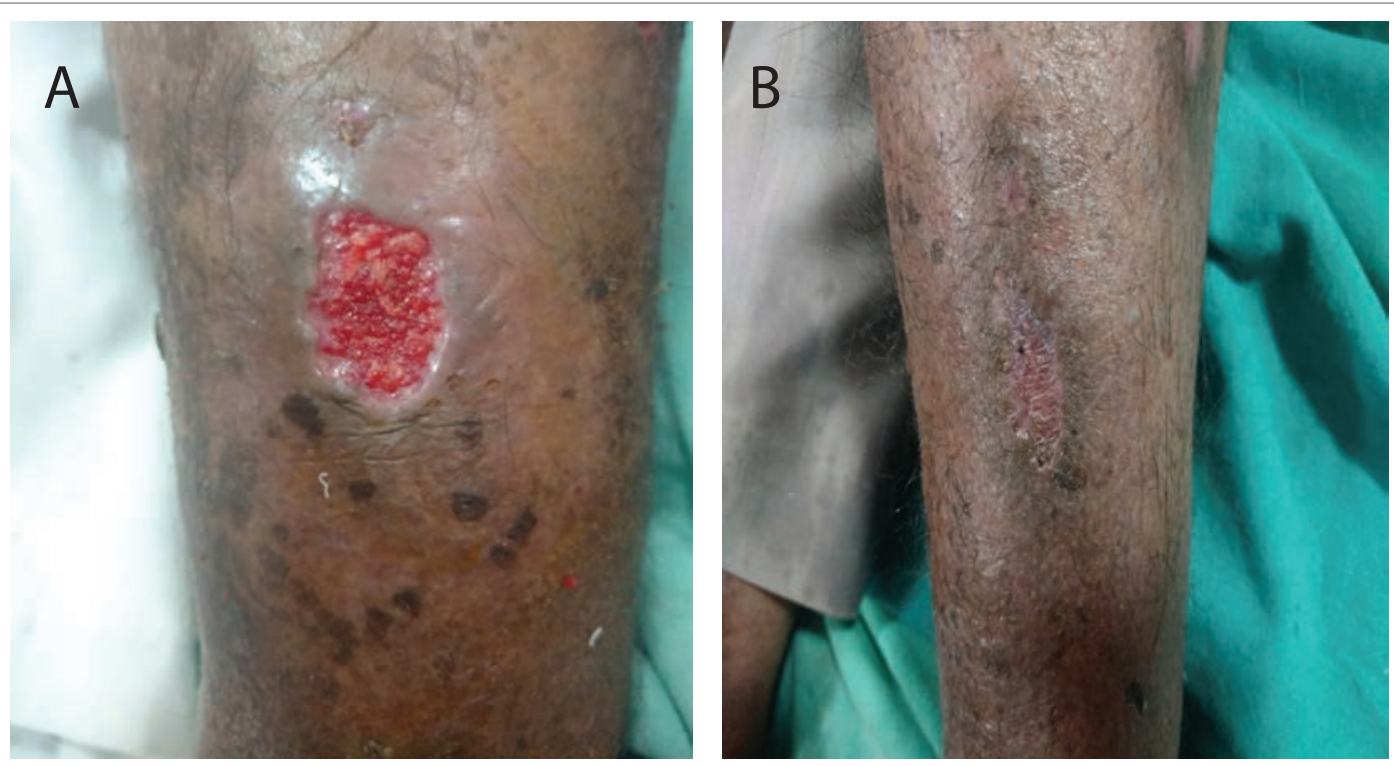

Figure 3: Long standing Diabetic ulcer on left lower leg.

A: Pre-procedure.

B: 8 weeks after the procedure.

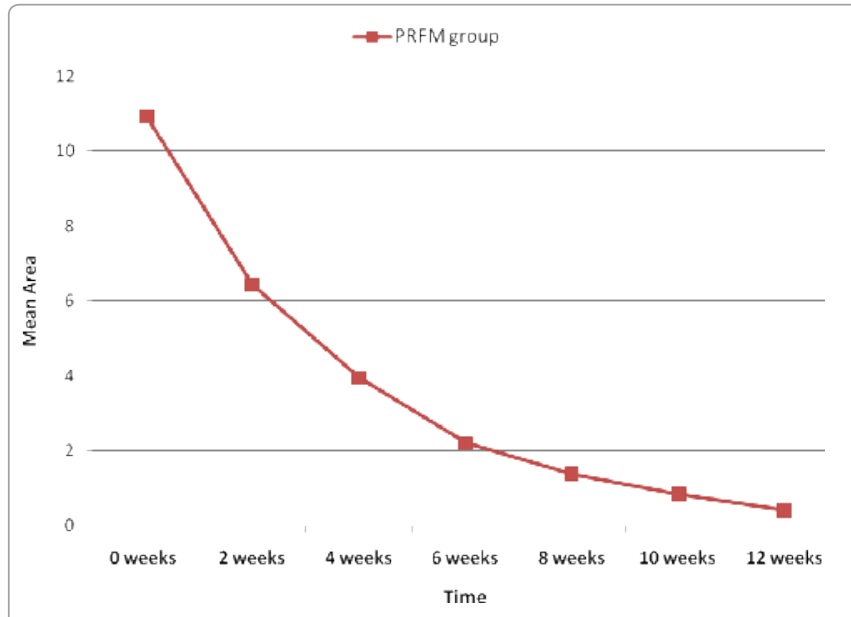

Figure 4: Change in the mean area of the ulcer.

architecture is responsible for a slow but sustained release of growth factors and matrix glycoproteins during initial $\geq 7$ days.

By localizing platelets and GFs and fostering a physiologic tissue response in the treated area, PRFM produces guided tissue regeneration [13].

25 patients were enrolled in our study and administered weekly sessions of Autologous PRFM in accordance to ulcer healing up to a maximum of 5 sittings. Reduction of pain and discharge from ulcer was noted in each patient beginning from the first session and all patients responded to the treatment in terms of reduction in the area and volume of ulcer. The mean time to ulcer healing was $6.43+2.33$ weeks.

In a study conducted by Suthar M, et al. [14] on the efficacy of PRP in chronic NHU the mean time to ulcer healing was found to be

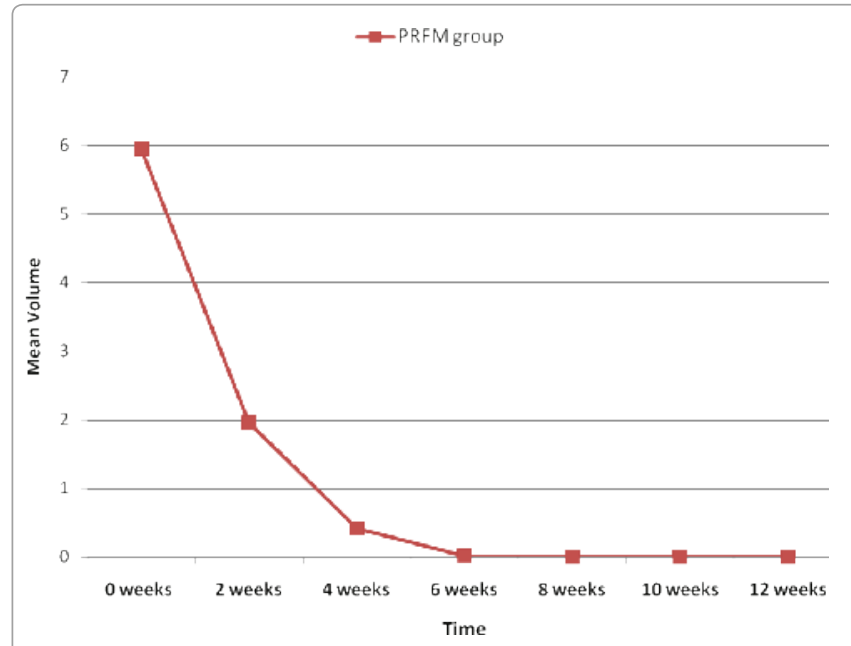

Figure 5: Change in the mean volume of the ulcer.

8.2 weeks. In a study conducted by Nagaraju U, et al. [15], the mean percentage of reduction in area and volume of the ulcers was $91.7 \%$ and $95 \%$ respectively at the end of 6 weeks.

In our study the mean time to ulcer healing was $6.43+2.33$ weeks and thee mean percentage of reduction in area and volume of the ulcers was $79.7 \%$ and $99.7 \%$ respectively at the end of 6 weeks.

In a similar study conducted by Nagaraju U, et al. [15] on 9 trophic ulcers in treated Hansen's disease patients the mean treatment period was 4.2 weeks. However, our study comprised of larger sample size and NHU of varying aetiology were included in our study.

\section{Conclusion}

Currently, there is no consistently effective treatment for NHU. This study has reviewed the potential of Autologous PRFM technique in the 
management of long standing NHU in an adult population. Following treatment of 25 patients, complete resolution of ulcers after twelve weeks was observed in 23 (92\%) patients while partial healing was observed in $2(8 \%)$ patients. Overall the mean time to ulcer healing was $6.43+2.33$ weeks. This technique is simple, easy to perform, economical, can be done within a restricted clinical setting and at the same time highly effective. However, a large scale multicentre study would provide a more detailed picture of efficacy of this promising technique.

\section{Conflict of Interest}

None

\section{Source(s) of Support}

None

\section{References}

1. Mekkes JR, Loots MA, Van Der Wal AC, Bos JD (2003) Causes, investigation and treatment of leg ulceration. $\mathrm{Br} J$ Dermatol 148: 388-401.

2. Iqbal A, Jan A, Wajid MA, Tariq S (2017) Management of Chronic Non-healing Wounds by Hirudotherapy. World J Plast Surg 6: 9-17.

3. Budamakuntla L, Loganathan E, Sarvajnamurthy SA, Nataraj HV (2017) Follicular unit grafting in chronic nonhealing leg ulcers: A clinical study. J Cutan Aesthet Surg 10: 200-206.

4. Dohan DM, Choukroun J, Diss A, Dohan SL, Dohan AJ, et al. (2006) Platelet-rich fibrin (PRF): A second-generation platelet concentrate. Part II: Platelet-related biologic features. Oral Surg Oral Med Oral Pathol Oral Radiol Endod 101: e45-e50.

5. Margolis DJ, Kantor J, Berlin JA (1999) Healing of diabetic neuropathic foot ulcers receiving standard treatment. A meta-analysis. Diabetes Care 22: 692-695.
6. Blume PA, Walters J, Payne W, Ayala J, Lantis J (2008) Comparison of negative pressure wound therapy using vacuum-assisted closure with advanced moist wound therapy in the treatment of diabetic foot ulcers: A multicenter randomized controlled trial. Diabetes Care 31: 631-636.

7. Marx RE, Carlson ER, Eichstaedt RM, Schimmele SR, Strauss JE, et al. (1998) Platelet-rich plasma: Growth factor enhancement for bone grafts. Oral Surg Oral Med Oral Pathol Oral Radiol Endod 85: 638-646.

8. Petrungaro PS (2001) Using platelet-rich plasma to accelerate soft tissue maturation in esthetic periodontal surgery. Compend Contin Educ Dent 22: 729-732, 734, 736.

9. Anitua E, Andia I, Ardanza B, Nurden P, Nurden AT (2004) Autologous platelets as a source of proteins for healing and tissue regeneration. Thromb Haemost 91: 4-15.

10. Choukroun J, Adda F, Schoeffler C, Vervelle A (2001) The opportunity in perio-Implantology: The PRF. Implantodontie 42: 55-62.

11. O'Connell SM, Impeduglia T, Hessler K, Wang XJ, Carroll RJ, et al. (2008) Autologous platelet-rich fibrin matrix as cell therapy in the healing of chronic lower-extremity ulcers. Wound Repair Regen 16: 749-756.

12. Yazawa M, Ogata H, Nakajima T, Mori T, Watanabe N, et al. (2003) Basic studies on the clinical applications of platelet-rich plasma. Cell Transplant 12: 509-518.

13. Sclafani AP (2011) Safety, Efficacy, and Utility of platelet-rich fibrin matrix in Facial Plastic Surgery. Arch Facial Plast Surg 13: 247-251.

14. Suthar M, Gupta S, Bukhari S, Ponemone V (2017) Treatment of chronic non-healing ulcers using autologous platelet rich plasma: a case series. J Biomed Sci 24: 16.

15. Nagaraju U, Sunder P, Agarwal P, Raju BP, Kumar M (2017) Autologous platelet-rich fibrin matrix in non-healing trophic ulcers in patients with Hansen's disease. J Cutan Aesthet Surg 10: 3-7. 